\title{
Joint Placement Latency Optimization of the Control Plane
}

\author{
Kurdman Abdulrahman Rasol Rasol \\ Department of Computer Architecture \\ Universitat Politècnica de Catalunya (UPC) \\ Barcelona, Catalonia, Spain \\ kurdman.rasol@upc.edu
}

\author{
Prof. Jordi Domingo-Pascual \\ Department of Computer Architecture \\ Universitat Politècnica de Catalunya (UPC) \\ Barcelona, Catalonia, Spain \\ jordi.domingo@ac.upc.edu
}

\begin{abstract}
Software Defined Networking (SDN) is a programmable network architecture that decouples the control plane (controller logic) from the data plane (forwarding plane) and provides flexible network management. A logically centralized control plane supports SDN feasibility. A single controller may not be capable to control the whole network. To tackle this issue, deploying multiple controllers are a necessity to handle a large network. Controller placement problem (CPP) is one of the most important issues in SDN to improve the scalability and influences the latency and performance of the whole network. Most of the previous methods only focused on latency between controllers and their associated switches but ignoring the latency between controllers and the accumulated latency of the control plane.

In this paper, we define an accumulated latency to solve CPP, which takes into consideration both the latency between the controller and their associated switches and inter-controller latency. We formulate an optimization problem as a mixedinteger linear programming (MILP) under the constraint of latency. The objective of our study is to minimize the accumulated latency and minimizing the number of network controllers while optimizing their placement for achieving an optimal balance at the same time. The performance of our method is evaluated on the Internet2 OS3E real network topology. Results demonstrate that the proposed method is promising.

Index Terms-Software Defined Networking, Controller Placement, Control Plane Latency, Mixed-Integer Linear Programming.
\end{abstract}

\section{INTRODUCTION}

Unlike traditional networks where both control plane and data plane are combined within a single networking device, the Software Defined Networking (SDN) architecture achieves highly programmability of the control plane (controller logic) by separating it from the data plane (forwarding plane) [1,2]. The data plane is responsible for forwarding network traffic, whereas the control plane manages the traffic. The control plane is responsible for high-level decision making and configuration for the data plane.

In distributed SDN controllers, two control planes are identified. First, we consider the control traffic exchanged between controllers and their associated switches indicated as (Ctr$\mathrm{Sw}$ ) plane. Second, we focus on the control traffic exchanged among controllers (or inter-controller) referred to as CtrCtr plane, which supports the interaction between controllers (i.e., the control traffic to keep the shared data structures synchronized). We focus our research on the latency tradeoff achievable in the Ctr-Sw and in the Ctr-Ctr control planes. The interaction between $\mathrm{Ctr}-\mathrm{Sw}$ plane corresponds to the southbound interface,i.e., OpenFlow, and the east-west interface corresponds to the interaction between Ctr-Ctr plane and the data stores.

For the communication within the control plane, the intercontroller traffic plane is important to achieve a consistent shared view of the network state, which is the essential requirement to run network applications correctly and maintain the network state that is stored in shared data structures. Reducing inter-controller latencies is necessary as reducing switches and controllers latencies but for topological reasons, reducing one kind of latencies implies maximizing the other and vice versa. The placement of the controller influences the total control traffic latency. Deploying multiple controllers increases the controller to controller traffic, while few concentrated controllers increase the controller to switch traffic latency.

The Ctr-Sw traffic refers to the number of packets transferred between the controller and their associated switches or from the switch to the controller. Moreover, the reaction time of sending the messages periodically from the controller to each switch traffic is usually shorter than the Ctr$\mathrm{Ctr}$ traffic communication. In general, Ctr-Ctr traffic is very complex because controllers may need to synchronize through a consensus algorithm shared data structures to guarantee a consistent global network view of the underlying network and to enable a centralized view of the network state for the applications. Then, the south-bound interface (Ctr-Sw traffic) communication is faster than the east-west interface (Ctr-Ctr traffic) communication.

The optimal placement of the controllers must not only consider the latencies of Ctr-Sw traffic, but also the latencies between controllers. Depending on the application of the control plane if is more important to minimize the Ctr-Ctr latency than the $\mathrm{Ctr}-\mathrm{Sw}$ traffic communication.

For each application running on the controller plane, we can modify the weight between $\mathrm{Ctr}-\mathrm{Sw}$ and $\mathrm{Ctr}-\mathrm{Ctr}$ latencies to determine the optimal placement of the controllers. Depending on the control plane application more weight for minimizing (inter-controller traffic or switch-controller traffic).

This work considers the joint optimization of latency and 
controller placement both the $\mathrm{Ctr}-\mathrm{Sw}$ (south-bound) interface and $\mathrm{Ctr}-\mathrm{Ctr}$ (east-west) interface. The main objective of this paper is to minimize the control plane latency, which takes into consideration both latency from the controller to switch and controller to the controller simultaneously.

The remainder of the paper is structured as follows. The related work in the Controller Placement Problem (CPP) is presented in Section II. The Mathematical Model and Formulation is described in Section III. The evaluation results are discussed in Section IV. Finally, in Section V we draw our conclusions.

\section{RELATED WORK}

In this section, we briefly discuss the most related works that addressed the controller placement problem in SDN. The controller placement problem (CPP) is a key design choice of the SDN control plane to increase performance. The problem is similar to the facility location problem, which can be denoted as non-deterministic polynomial NP-hard problem [3]. Heller et al [4] firstly improve the scalability of multicontroller for solving CPP to determine the optimum location of the controllers deployed and the minimal number of controllers to be placed. The authors on the Internet2 OS3E topology evaluate the performance [5]. The placement of the controller directly influences the latency between switches and associated controllers, thus affecting the performance of the whole network. To solve the CPP, the authors propose an optimized algorithm based on the K-means and K-centre algorithm. K-means algorithm is designed for minimizing the average propagation delay between the controllers and their assigned switches [6], while K-center is designed to minimize the maximum latency between controllers and switches [7]. Earlier solutions of CPP only focused on propagation latency between controllers and their associated switches and intercontroller latency metrics were ignored.

Hock et al. [8] proposed a framework for resilient Paretobased Optimal Controller Placement (POCO) that provides the operator of a network with all Pareto-optimal placements. In their paper, the load on the controllers and inter-controller latency is considered. The POCO provides better load balancing between switches and its controllers and balanced the placement of controllers. If a large number of controllers are required for managing the network, synchronization is necessary to maintain a consistent global state. Depending on the frequency of the inter-controller synchronization, the latency among controllers plays an important role and thus should be considered during the controller placement.

Gao et. al. [9] authors introduce a framework for the Global Latency Control Placement Problem with Capacitated Controllers (CGLCPP). Global latency is the combination of the switch to controller latency and inter-controller latency. The optimization objective is to minimize global latency on random network topologies, which takes into consideration the latency between controllers and switches, the latency between controller to controller and the capacities of controllers. In their theoretical research paper, they proposed a Particle Swarm
Optimization (PSO) algorithm to solve the control placement in SDN but they did not consider the reliable placement of controllers (RCPP). The PSO-based solution provides better performance in terms of latency and computational time as compared to the integer linear programming (ILP) and greedy algorithms.

Wang et al. [10] implement Network Clustering-based Particle Swarm Optimization Algorithm (NCPSO) to optimize the controller load under propagation delay and load-balancing constraints. The authors extended the previous work of Gao et al [9]. Moreover, they divide the network into $\mathrm{k}$ small domains where each domain has its own controller for controlling and management. In their paper, the load of controllers, switch to controller latency, inter-controller latency, and load balancing are considered. Their simulation results show the effectiveness of the proposed algorithm can significantly reduce the number of required controllers, reduce the load of the maximum-load controller and load balance. They randomly generated some different size of topology. Results show better performance as compared to K-center and capacitated K-center strategy $[4,11]$.

The authors of [12] defined the reliable controller placement problem (RCPP) with the implementation of PSO (particle swarm optimization) algorithm to solve CPP and TLBO (teaching-learning based optimization) to solve the RCPP in the network. The objective is to minimize the total average latency of a reliable network. In their paper, the simulations are tested for two most popular topologies (Internet2 with 34 nodes) and (Savvis with 19 nodes). More practically, simulation results show that the solution of TLBO gives better performance as compared to PSO based solution for CPP.

In our study, we define a new metric for the joint optimization of latency taking into account the Ctr-Sw latency and $\mathrm{Ctr}-\mathrm{Ctr}$ latency simultaneously. The objective of this study is to find the optimal placement of the controllers that minimizes the accumulated latency between Ctr-Sw and CtrCtr. As well in this work, we focus exclusively on minimizing the number of controllers required while optimizing their placement between $\mathrm{Ctr}-\mathrm{Sw}$ latency and $\mathrm{Ctr}-\mathrm{Ctr}$ at the same time. Further studies are needed to consider the placement problem for including reliability.

\section{Formulation of Joint Placement LATENCY OPTIMIZATION}

The SDN network topology can be represented by a graph $G=(V, E)$, where $V$ represents the set of all switches (or nodes) and $E$ represents the set of all physical edges (or links) between the switches, where the link weight refers to physical distance.

We denote the set of switches as $V=\left\{v_{1}, v_{2}, \ldots, v_{n}\right\}$, where $n$ represents the total amount of nodes $(n=|V|)$. The set of controllers to be installed is represented as $C=$ $\left\{c_{1}, c_{2}, c_{3}, \ldots, c_{k}\right\}$, where $k$ represents the number of controllers to be deployed throughout the network $(k \leq n)$. Let $M$ denote the distance matrix between all edges and $\operatorname{dist}_{(i, j)}$ denote the distance from the node $i$ to node $j$. A node may 
TABLE I

NOTATIONS AND DEFINITIONS.

\begin{tabular}{||cc||}
\hline \multicolumn{1}{||l||}{ NOTATIONS } & Definition \\
\hline \hline$G(V, E)$ & Graph G, where V is a set of switches and E is set of edges between switches \\
\hline$V$ & Set of switches or nodes in the network \\
\hline$E$ & Set of physical links or edges between switches \\
\hline$C$ & Set of Controllers to be installed, where $(C \subset V)$ \\
\hline$M$ & A matrix consisting of the shortest path latency. For each index $(i, j)$, it corresponds to the shortest path latency from node $i$ to node $j$. \\
\hline$d i s t t_{(i, j)}$ & the distance from the node $i$ to node $j$ \\
\hline$(i, j)$ & Link from node $i$ to node $j$ \\
\hline$n$ & Total number of nodes in the network, where $(n=|V|)$ \\
\hline$k$ & Indicate whether switch $i$ is mapped to controller $j(=1)$ or not $(=0)$ \\
\hline$x_{i, j}$ & Denotes whether controller $k$ is placed onto switch $i(=1)$ or not $(=0)$ \\
\hline$y_{i, j}$ & The number of possible links between controllers is $k(k-1) / 2$ \\
\hline$n_{(C t r-C t r)}$ & Controller-Switch latency \\
\hline$C t r-S w$ & Controller-Controller latency (or Inter-controller latency) \\
\hline$C t r-C t r$ &
\end{tabular}

be a simple switch or a controller collocated with the switch. The details of the notation is summarized in Table I.

Joint placement latency optimization model is based on the original CPP model [4] with some additional constraint and terms for the objective function. More particularly, we model the joint placement latency optimization to find an optimal trade-off between switch-controller latency and inter-controller latency. The goal is to minimize the accumulated latency of the network, mathematically formulated as:

$$
\min \left(\lambda z_{1}+(1-\lambda) z_{2}\right)
$$

The first part of the objective function considers the latency between switches and controller $(z 1$ weighted by $\lambda)$ and the second part of function considers the inter-controller latency ( $z 2$ weighted by $(1-\lambda)$ ). In other words, the accumulated latency is the weighted combination of switch to controller latency and inter-controller latency. The objective is to determine the optimal number and location of controllers to achieve a given balance between controllers and switches latency, and controller to controller latency in the control plane.

In this work, we consider the latency as the distance by using Dijkstra's algorithm for computing the shortest path distance between pairs of nodes. Furthermore, we consider two types of optimization metrics: average-case latency and worst-case latency. The controller is collocated with a switch and each switch is managed by a single controller.

1) The latency between controllers and switches (Ctr Sw):

- Average-case latency scenario.

The average latency between controllers and their assigned switches is computed as:

$$
z_{1}=\frac{\sum_{i \in V, j \in C} \operatorname{dist}_{(i, j)} x_{i, j}}{(n-k)}
$$

Where $\operatorname{dist}_{(i, j)}$ is the shortest distance path from switch $i$ to controller $j$ and $n$ represents the total amount of nodes in the network and $k$ represents the number of the controllers. This optimization problem is known as the minimum k-means problem.

- Worst-case latency scenario.

The objective is to minimize the maximum latency between the controller and their associated switches.

$$
z_{1}=\max _{j \in C}\left(\operatorname{dist}_{(i, j)} x_{i, j}\right), \forall i \in V, j \in C
$$

This optimization problem is known as minimum k-center problem.

2) Inter-controller communication latency $(\mathrm{Ctr}-\mathrm{Ctr})$ :

- Average-case latency scenario.

The average inter-controller latency is minimized. The number of possible paths among controllers highly dependent on number of controllers $k$ is calculated as $n_{(C t r-C t r)}=k(k-1) / 2$. In order to compute the average latency, the following equation is used:

$$
z_{2}=\frac{\sum_{i \in V, j \in C} \operatorname{dist}_{(i, j)} x_{i, i} y_{j, j}}{n_{(C t r-C t r)}}
$$

- Worst-case latency scenario.

To solve the worst-case inter-controller latency, the longest distance between controllers is computed. The optimization problem is to minimize the maximum latency among controllers. The considered metric is formulated as:

$$
z_{2}=\max _{j \in C}\left(\operatorname{dist}_{(i, j)} x_{i, i} y_{j, j}\right), \forall i \in V, j \in C
$$

The constraints of this model are formulated as:

$$
\begin{aligned}
& \sum_{j \in C} x_{i, j}=1, \forall i \in V \\
& \sum_{i \in V} y_{i, j}=1, \forall j \in C \\
& y_{i, j} \leq x_{i, j}, \forall i \in V, \forall j \in C \\
& x_{i, j}, y_{i, j} \in\{0,1\}
\end{aligned}
$$

Constraint (6) guarantees that every switch is assigned to exactly one controller. Constraint (7) ensures that each controller 


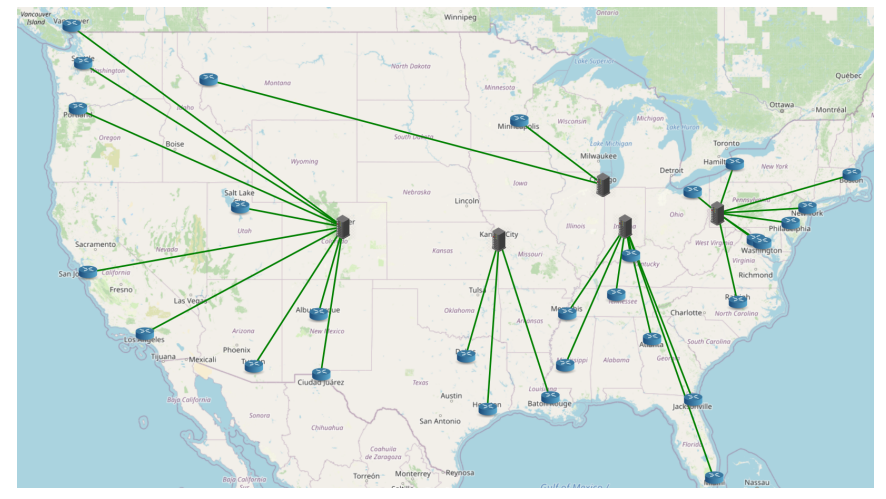

Fig. 1. The optimal controller location that provides a balance between average switch to controller latency and average inter-controllers latency $(\lambda$ $=0.8$ and $k=5$ )

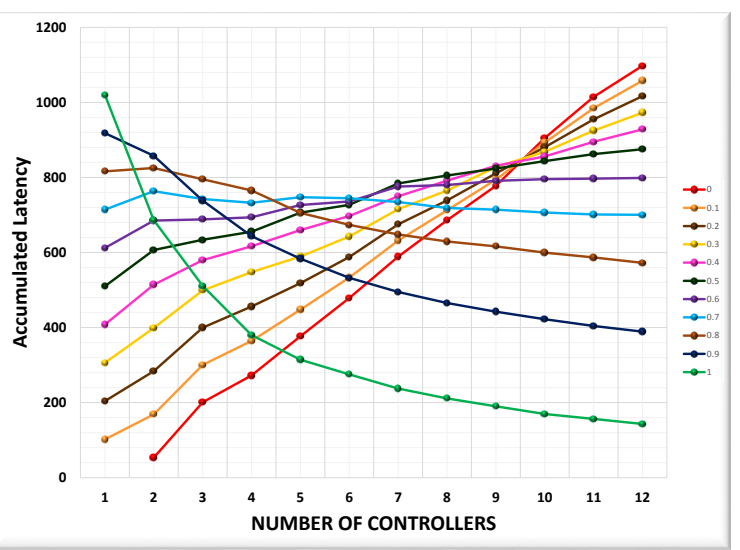

Fig. 2. The Accumulated Latency of average switch to controller latency and average inter-controllers latency

is located onto exactly one switch. Constraint (8) ensures that switch $i$ is mapped to the controller $j$ if controller $j$ is placed onto switch $i$. Constraint (9) guarantees that the decision variables are binary (equal to 0 or 1 ).

\section{Evaluation Results}

The goal of the model is to minimize the accumulated latency of the network, which takes into consideration the latency between controllers-switches and inter-controller simultaneously. Therefore, the placement of controllers may vary according to the parameter $\lambda$. The real network topology used is Internet2 OS3E network, which has 34 nodes and 42 links. The mathematical models are implemented in Python. We run the optimization as a mixed-integer linear programming (MILP) model. Then, we solve the problem by using Gurobi Optimizer. The Gurobi Optimizer is one of the fastest and most powerful solver.

\section{A. Sensitivity analysis of lambda}

When the weight of $\lambda$ increases, the objective function is more focused on the latency between controllers and their associated switches and when the $\lambda$ value decreases the objective function is more focused on the inter-controller communication latency.

Figure 1 shows how different values of $\lambda$ influence the optimal placement of the controllers for 5 controllers. The placement of the controllers in the network is of paramount importance and implies different trade-offs between the switch to controller latencies and controller to controller latencies.

Figure 2 depicts the results of the accumulated latency of average switch-controller latency and average Inter-controller latency when the number of controllers varies from 1 to 12 and the values of $\lambda$ range from 0 to 1 .

When the weight of $\lambda$ is one, we optimize the results for the accumulated latency between switches and its controllers only. This case corresponds with the basic CPP optimization [4]. Each switch selects the nearest controller in the network. For 5 controllers, the optimal average latency is 314.44 miles and the optimal placement of the controllers is Seattle, El Paso (Texas), Nashville, Houston and Ashburn (Virginia). Results show that the average latency between controllers and switches can be reduced by increasing the number of controllers.

When the value of $\lambda$ is zero, the optimization considers the inter-controller latency only and all the controllers are at the closest distance. In this case, because the model does not consider the switches and controllers latency some switches might be far from their assigned controller. Increasing the number of controllers, the accumulated latency sharply increases. For 5 controllers, the optimum accumulated latency is 376.93 miles and the controller location is New York, Philadelphia, Washington DC, Pittsburgh and Ashburn (Virginia).

When the value of $\lambda$ is 0.5 means that a balance between controller-switch latency and inter-controller latency is sought. For 5 controllers, the optimal placement of controllers is Chicago, Indianapolis, Louisville, Nashville and Memphis and yield minimum accumulated latency is 706.14 miles.

When the number of controllers is 5 and $\lambda=0.8$, the minimum accumulated latency is 705.46 miles and the optimal controller location is Denver, Chicago, Kansas City (Missouri), Indianapolis and Pittsburgh (see Figure 1).

It is worth to note that, when the number of controllers deployed reaches a certain value in the network, the accumulated latency begins to increase as the number of controllers increases. This is because when the number of controllers increases, the inter-controller latency increases rapidly.

This method can be applied under different scenarios: average Ctr- $\mathrm{Sw}$ latency and worst-case Ctr-Ctr latency, worstcase $\mathrm{Ctr}-\mathrm{Sw}$ latency and average $\mathrm{Ctr}-\mathrm{Ctr}$ latency, worst-case $\mathrm{Ctr}-\mathrm{Sw}$ latency and worst-case Ctr-Ctr latency.

\section{B. Balancing switch-controller and inter-controller latency}

In the first analysis, we define the weight for controllers to switches and Inter-controllers by setting the value of $\lambda$. For each control plane application a value of $\lambda$ is set and the placement of the minimum number of controllers can be found. In this subsection, we assume that a balance between 
TABLE II

THE DIFFERENCE BETWEEN AVERAGE SWITCH-CONTROLLER LATENCY AND AVERAGE INTER-CONTROLLER LATENCY (MILES)

\begin{tabular}{|l|l|l|l|l|l|l|l|l|l|}
\hline $\mathrm{k} / \lambda$ & 0.1 & 0.2 & 0.3 & 0.4 & 0.5 & 0.6 & 0.7 & 0.8 \\
\hline \hline $\mathrm{k}=2$ & 1152.33 & 1152.33 & 1152.33 & 1152.33 & 787.77 & 787.77 & 617.91 & 617.91 & 6 \\
\hline $\mathrm{k}=3$ & 993.89 & 993.89 & 993.89 & 542.93 & 542.93 & 542.93 & 542.93 & 283.83 & 1984.43 \\
\hline $\mathrm{k}=4$ & 917.52 & 917.52 & 917.52 & 387.32 & 387.32 & 387.32 & 387.32 & 959.27 & 1422.59 \\
\hline $\mathrm{k}=5$ & 707.65 & 707.65 & 707.65 & 707.65 & 206.77 & 206.77 & 206.77 & 885.34 & 2020.52 \\
\hline $\mathrm{k}=6$ & 547.01 & 547.01 & 547.01 & 547.01 & 87.31 & 87.31 & 87.31 & 836.23 & 2010.37 \\
\hline $\mathrm{k}=7$ & 431.2 & 431.2 & 342.48 & 342.48 & 342.48 & 330.66 & 794.1 & 1021.42 & 2032.83 \\
\hline $\mathrm{k}=8$ & 263.98 & 263.98 & 263.98 & 263.98 & 24.54 & 452.8 & 831.77 & 943.8 & 1951.05 \\
\hline $\mathrm{k}=9$ & 166.99 & 166.99 & 166.99 & 73.39 & 73.39 & 506 & 922.62 & 985.43 & 2229.56 \\
\hline $\mathrm{k}=10$ & 123.2 & 123.2 & 123.2 & 123.2 & 123.2 & 577.75 & 968.93 & 1389.28 & 2218.67 \\
\hline $\mathrm{k}=11$ & 299.1 & 299.1 & 299.1 & 299.1 & 432.08 & 905.02 & 1021.02 & 1386.9 & 2103.24 \\
\hline $\mathrm{k}=12$ & 396.2 & 443.09 & 443.09 & 443.09 & 674.48 & 963.77 & 1020.42 & 1703.81 & 2055.05 \\
\hline
\end{tabular}

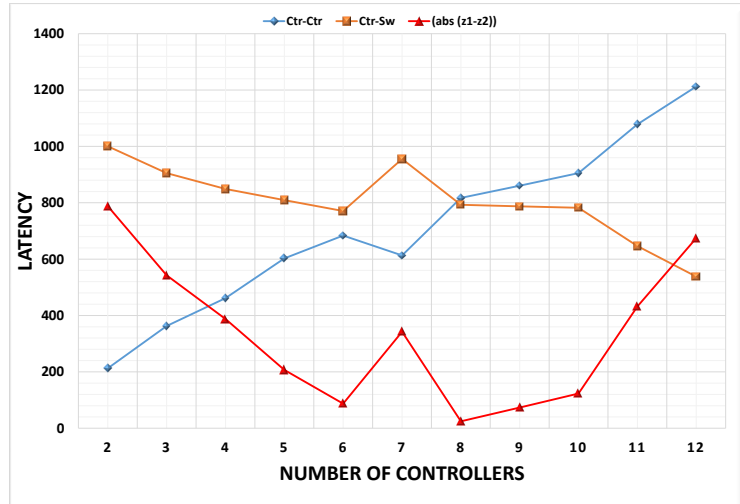

Fig. 3. The average switch to controller latency and average inter-controller latency $(\lambda=0.5)$

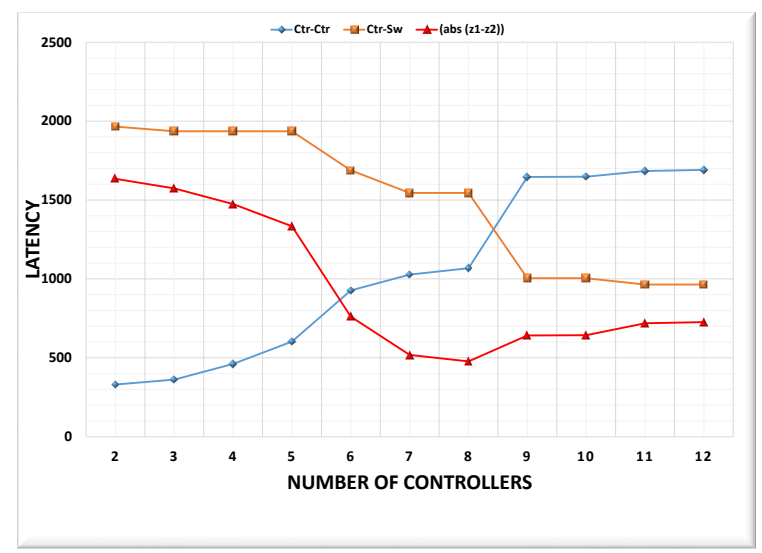

Fig. 5. The worst-case switch to controller latency and average intercontroller latency $(\lambda=0.5)$

$\mathrm{Ctr}-\mathrm{Sw}$ and $\mathrm{Ctr}-\mathrm{Ctr}$ latency is also a goal to achieve the required performance of the control plane.

The next step in our work, we define a decision rule that provides a balance between controllers and its associated switches and inter-controllers simultaneously. Table II shows the difference between $\mathrm{Ctr}-\mathrm{Sw}$ and $\mathrm{Ctr}-\mathrm{Ctr}$ latency expressed

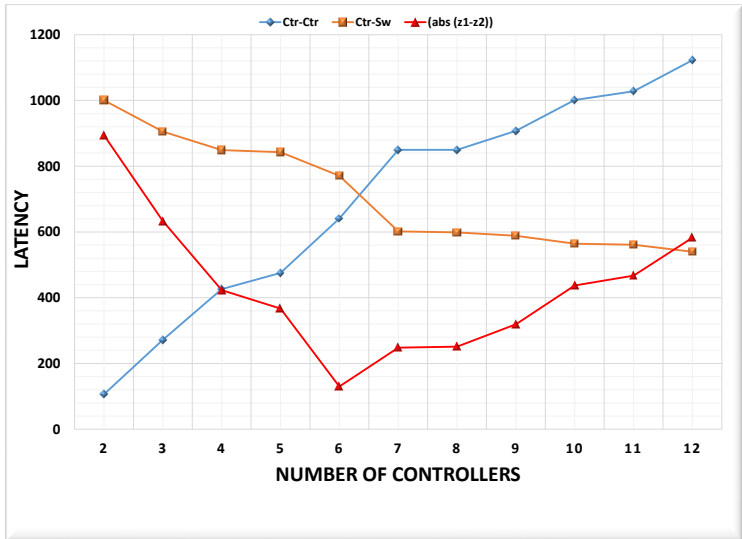

Fig. 4. The average switch to controller latency and worst-case intercontroller latency $(\lambda=0.5)$

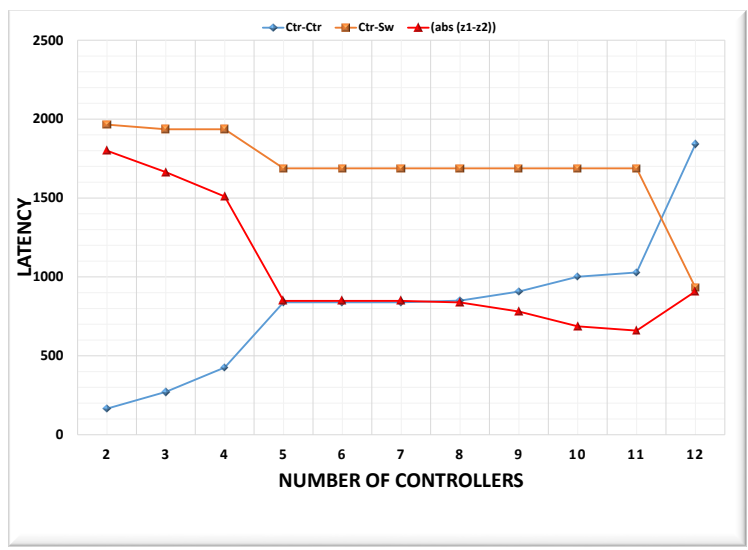

Fig. 6. The worst-case switch to controller latency and worst-case intercontroller latency $(\lambda=0.5)$

as:

$$
(a b s(z 1-z 2))
$$

Figures 3, 4, 5 and 6 depict the comparison results of the overall network latency with the minimum number of controllers that provides the tradeoff between Ctr-Sw latency and Ctr-Ctr latency to achieve a balance when the number of controllers 


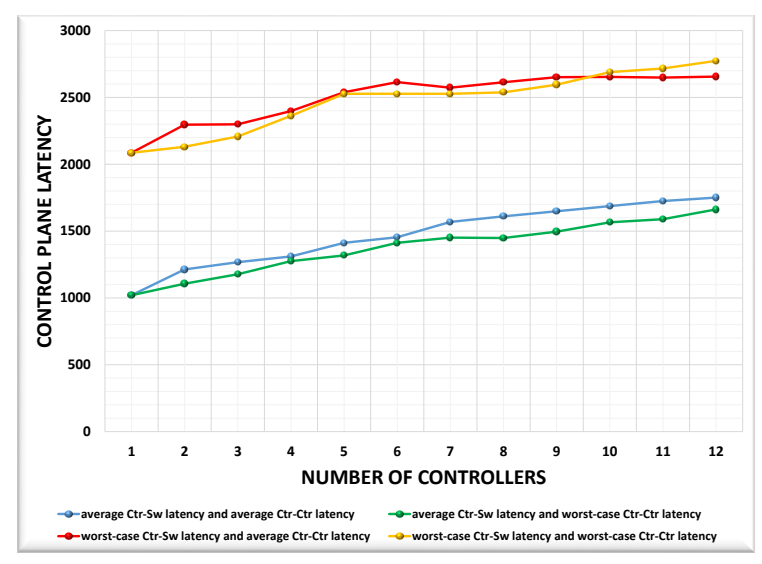

Fig. 7. The control plane latency in Internet2 OS3E topology

varies from 2 to 12 and $\lambda=0.5$. We consider the impact of the number of controllers on the latency performance. The latency between $\mathrm{Ctr}-\mathrm{Sw}$ decreases with more controllers in the network while the latency among controllers increases with the increase of the number of controllers.

Figure 3 depicts the average Ctr-Sw latency and average Ctr-Ctr latency with a different number of controllers. In this case, the balance is achieved with 8 controllers. In the case of the average $\mathrm{Ctr}-\mathrm{Sw}$ latency and worst-case $\mathrm{Ctr}-\mathrm{Ctr}$ latency, 6 controllers are sufficient to manage the control plane scalability (see Figure 4). Figure 5 shows the impact of the number of controllers on the worst-case Ctr-Sw latency and average Ctr-Ctr latency. Results show the latency tradeoff achievable for 8 controllers to perform better performance. Figure 6 shows the result of worst-case Ctr-Sw latency and worst-case Ctr-Ctr latency. In this case, the optimum number of controllers deployed on the best locations is 11 controllers. Thus, the proposed method is applicable to all other cases with different values of $\lambda$ and average and worst-case latency.

\section{The control plane latency}

In this subsection, we define the control plane latency as a sum of Ctr-Sw and Ctr-Ctr latency. As illustrated in Figure 7 , the average-case latency based solution gives better results as compared to the worst-case latency based solution for the placement of controllers in the control plane. It is interesting to note that average $\mathrm{Ctr}-\mathrm{Sw}$ gives always a better performance than considering the worst-case scenario. Instead, the worstcase scenario might be useful for Ctr-Ctr latency optimization.

\section{CONCLUSION}

Placing multiple controllers is an essential requirement for managing large-scale networks. In this paper, we consider the accumulated latency to solve the placement of controllers, which determines the number of required controllers and the location of the controllers to achieve a joint optimization between the controller and its associated switches as well as inter-controller communications. We conduct the experiments on Internet2 OS3E real network topology. We propose that depending on the control plane applications a value for lambda will be selected. Running different applications in the control plane may require different values of $\lambda$. According to the value of $\lambda$, we provide the solution to determine the optimal placement of the controllers for each case. The results show that as the number of controllers increases and the placement of controllers becomes more distributed, the controllers get closer to the switches and the latency of the $\mathrm{Ctr}-\mathrm{Sw}$ decreases. On the other hand, if the controllers are fewer and more concentrated, then the latency of the $\mathrm{Ctr}-\mathrm{Ctr}$ decreases. Once the value of lambda is set according to the control plane application, the optimal number of controllers to balance Ctr$\mathrm{Sw}$ and Ctr-Ctr latencies may be found. Finally, results show that average latency optimization is better than worst-case for Ctr-Sw.

\section{ACKNOWLEDGEMENTS}

The work was supported by the Spanish Ministry of Economy and Competitiveness under contract TEC2017-90034-C21-R (ALLIANCE).

\section{REFERENCES}

[1] Jammal M, Singh T, Shami A, Asal R, Li Y. Software defined networking: state of the art and research challenges. Computer Networks. 2014;72:74-98.

[2] Singh AK, Srivastava S. A survey and classification of controller placement problem in SDN. Int J Netw Manag. 2018;28(3). https://doi. org/10.1002/nem.2018

[3] Arya V, Garg N, Khandekar R, Meyerson A, Munagala K, Pandit V. Local search heuristics for k-median and facility location problems. SIAM J Comput. 2004;33(3):544-562.

[4] Heller B, Sherwood R, McKeown N. The controller placement problem. In: Proceedings of the First Workshop on Hot Topics in Software Defined Networks. Helsinki, Finland: ACM; 2012:7-12.

[5] F. Yeung, "Internet 2: scaling up the backbone for R\&D," in IEEE Internet Computing, vol. 1, no. 2, pp. 36-37, March-April 1997, doi:10.1109/4236.601096.

[6] G. Wang, Y. Zhao, J. Huang, Q. Duan and J. Li, "A K-means-based network partition algorithm for controller placement in software defined network," 2016 IEEE International Conference on Communications (ICC), Kuala Lumpur, 2016, pp. 1-6, doi: 10.1109/ICC.2016.7511441.

[7] K. S. Sahoo, B. Sahoo, R. Dash and M. Tiwary, "Solving Multicontroller Placement Problem in Software Defined Network," 2016 International Conference on Information Technology (ICIT), Bhubaneswar, 2016, pp. 188-192, doi: 10.1109/ICIT.2016.047.

[8] Hock, D., et al.: POCO-framework for Pareto-optimal resilient controller placement in SDN-based core network. In: IEEE NOMS (2014)

[9] Gao C, Wang H, Zhu F, Zhai L, Yi S. A particle swarm optimization algorithm for controller placement problem in software defined network. In: Proceedings of the International Conference on Algorithms and Architectures for Parallel Processing (ICA3PP 2015); 2015; Zhangjiajie, China.

[10] Liu S, Wang H, Yi S, Zhu F. Ncpso: a solution of the controller placement problem in software defined networks. Proceedings of the International Conference on Algorithms and Architectures for Parallel Processing (ICA3PP15). Zhangjiajie, China: Springer; 2015:213-225.

[11] Guang, Y., Jun, B., Yuliang, L., Luyi, G.: On the capacitated controller placement problem in software defined networks. IEEE Commun. Lett. 18, 1339-1342 (2014).

[12] Singh AK, Kumar N, Srivastava S. PSO and TLBO based reliable placement of controllers in SDN. Int $\mathbf{J}$ Comput Netw Inf Secur. 2019;11(2):36-42. 